\title{
Design and Implementation of Learning Process Tracking System based on SCORM
}

\author{
Xing-hua $\mathrm{Sun}^{1}$ li Hao ${ }^{1}$ Ze-xin $\mathrm{An}^{2}$ Jun-hua Liang ${ }^{1}$ \\ ${ }^{1)}$ School of Information Science and Engineering, Hebei North University, Zhangjiakou, Hebei, China \\ ${ }^{2)}$ First Affiliated Hospital, Hebei North University, Zhangjiakou, Hebei, China
}

\begin{abstract}
It has remained a difficult job to record and track the learning process of learners in online learning system. SCORM standard defines a powerful learning tracing mechanism, which can achieve timely, detailed, in-depth learning tracing. In this paper, the learning tracing theory of SCORM standard is analyzed and A solution of Learning Process Tracking System is propose. The technology related to the implementation is introduced, and the designs of every subsystem of the solution, as well as the implementation of its prototype, are introduced in details.
\end{abstract}

Keywords—scorm 2004,learning Process Tracking System, data Model, learning record

\section{基于 SCORM 标准的学习过程跟踪系统的设计与实现}

\author{
孙兴华 ${ }^{1}$ 郝丽 $^{1}$ 安泽心 $^{2}$ 梁俊花 $^{1}$ \\ 1) 河北北方学院信息科学与工程学院, 张家口, 河北, 中国 \\ 2) 河北北方学院附属第一医院, 张家口, 河北, 中国
}

摘 要 在线学习平台中, 跟踪和记录学习者的学习过程是一件非常困难的事情, SCORM 标准定义了很好的学习跟踪机制, SCORM 标准能及时详细的跟踪记录学习者的学习状态。本文分析了 SCORM 标准的学习跟踪理论, 提出了在线学习跟踪系统的解决方案, 阐 述了跟踪系统的实现技术, 并详细的阐述了系统的功能设计、实现原型等。

关键词 SCORM 2004, 学习过程跟踪系统, 数据模型, 学习记录

\section{1. 引言}

近年来, 随着 MOOCs、翻转课堂这些新型教学模式的 迅速兴起, E-Learning 发展的可谓如火如茶、遍地开花。然 而当前 E-Learning 在发展中也面临很多问题。无论是 MOOCs 还是翻转课堂, 学生在学习过程中的自主性增强, 教师的角色削弱。因此如果不能对学生的在线学习过程进 行有效的监督、记录, 将无法记录学生珍贵的学习数据, 也就 不能更好地指导、督促学员完成整个教学过程, 达到预期 的学习效果。如何对学生学习课件的过程进行有效的记录 和跟踪? 如何既要记录学员在线学习课件的学习过程, 也要 有效记录学生在线评价的结果, 是需要在线学习系统需要 考虑的重要问题。

课题研究了国内外的相关学习标准, 并对 SCORM 2004
标准作了深入的学习和探讨,在此基础上, 设计开发了符合 SCORM2004 标准的在线学习过程跟踪系统。

\section{SCORM 2004 概述}

SCORM 2004 ( Sharable Content Object Reference Model) 主要由三部分构成: 内容聚合模型（Content Aggregation Model, CAM)、运行时间环境 (Run-time Environment， RTE）和排序与导航 ( Sequencing and Navigation, SN)。

(1) SCORM 内容聚合模型 (CAM): CAM 的目的是提供一 个公共的方法, 把学习资源组合成学习内容。

(2) SCORM 运行时间环境 (RTE): SCORM 规范的目标之 一，就是希望能够实现学习资源在多个 LMS/LCMS 之

河北省高等教育教学改革研究与实践项目支持（资助号：2015GJJG138), 河北省卫生厅青年科技课题支持（20110171） 
间的重用性和互操作性。为了实现这个目标, 必须建立: 一个启动学习资源的通用方法; 一套通用的学习资源与 LMSLLCMS 之间的通信机制; 以及学习资源与 LMS/LCMS 之间所交换的标准数据集合。运行时间环 境的这三个方面分别对应运行(Launch)、应用编程接口 (API)和数据模型(Data Model)。

（3）数据模型(Data Model): 构建一个通用的数据模型, 有 两个目的: 一是建立学习资源和 LMS/LCMS 之间所交 换的标准数据集合; 二是定义与 SCO 对象相关的属性、 行为、关联、组合、继承等, 使得 SCO 能在不同 LMS/LCMS 平台上被跟踪。

\section{3. 系统设计}

包括系统功能设计、数据库设计和系统架构设计等内 容。

\section{1 系统的功能设计}

本系统实现的功能主要包括用户注册、课程管理、用 户管理、学习管理、课程学习等功能。系统总的用例图如 图 1 所示。

(1) 课程管理模块: 主要完成 SCORM 课程管理, 提供 SCORM 课程的导入、编辑以及删除等功能。SCORM 课程的导入不是一个简单的上传过程, 它需要对课程做 一系列的验证、分析工作, 为以后的学习过程做好必要 的准备。上传的课程在数据库中按照一定的规则加以存 储, 以便使用者能够检索和快速定位到所需要的学习对 象。

(2) 用户管理模块: 分为两级管理, 其中管理员可以添加用 户、删除用户、修改用户信息; 学生可以修改本人的信 息。

(3) 学习管理模块:可以设定各级学习目标, 并能查看系统 记录的学习者的学习状态。

(4) 学习过程跟踪模块: 学习过程跟踪模块是系统的核心模 块。一个完整的课程学习过程包括: 首先学生要注册欲 汶览的课程, 这个过程为学生进入该课程做一系列预处 理动作; 然后, 学生 就可以进入课程开始学习了, 系 统提供了多种学习方式, 一种学生可以根据 SCORM 预 先规定的序列学习; 一种是系统提供了强大的导航功 能, 学生可以依据个人需要通过这些学习导引机制学 习; 还有一种就是系统提供了学习对象的搜索功能, 学 习者只需输入关键字系统就会自动生成相应的课程。

(5) 系统维护模块: 用来维护系统的稳定性, 对系统以及课 程做一些初始化和清空的动作。

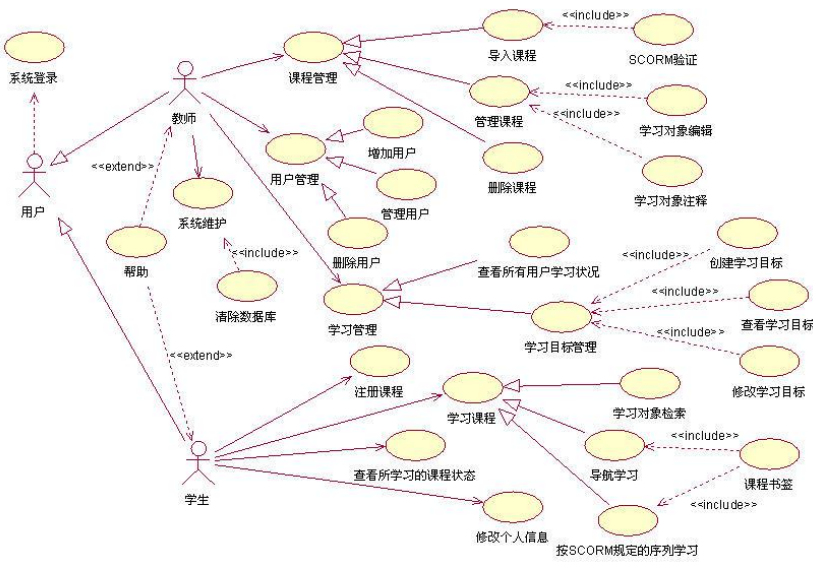

图 1 学习过程跟踪系统用例图

3.2 数据库的设计

根据 SCORM2004 标准, 为实现学习过程的跟踪记录, 主要设计如下数据表。

(1) UserInfo 表: 用来记录 LCMS 的注册用户的基本信息和 层级水平。

（2）CourseInfo 表: 存储 SCORM 课程的基本信息, 其中 Active 用于表示当前课程的共享状态。

(3) ApplicationData 表: 用来唯一标识上传 SCORM 课程的 计数器, 记录了已经上传了几门课程, 下一门课程的 编号是什么。

(4) CourseStatus 表: 用来记录学生对课程的学习情况和对 所学课程的满意程度。

(5) UserCourseInfo 表: 用来记录每个学生选课情况。

(6) ItemInfo 表: 存储 SCORM 课程中每个学习对象的详细 信息, 这些信息的获取是通过解析内容清单文件得到, 每一条记录都对应 imsmanifest.xml 中定义的一个 <item>项, 用来记录学习对象的详细信息, 包括它的标 题、类型、启动地址、元数据以及课程设计者对于该 学习对象的特殊教学约定。

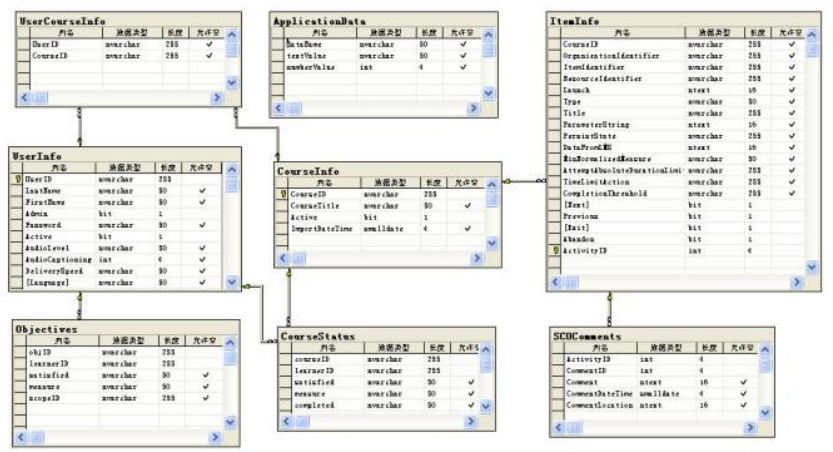

图 2 学习记录跟踪系统主要数据表设计 


\section{3 系统架构设计}

一个设计良好的系统框架对于应用系统的成败起着至 关重要的作用。本系统涉及到多个功能模块间数据的交流 和共享, 所以必须在分析设计阶段对整个系统有全局的掌 控, 这样做避免了开发过程中逻辑的混乱, 也使以后的维 护和升级扩展变得非常容易。从系统逻辑上, 笔者按照多 层体系架构的理念对整个系统进行了划分。图 3 为本学习 过程跟踪系统的系统框架。

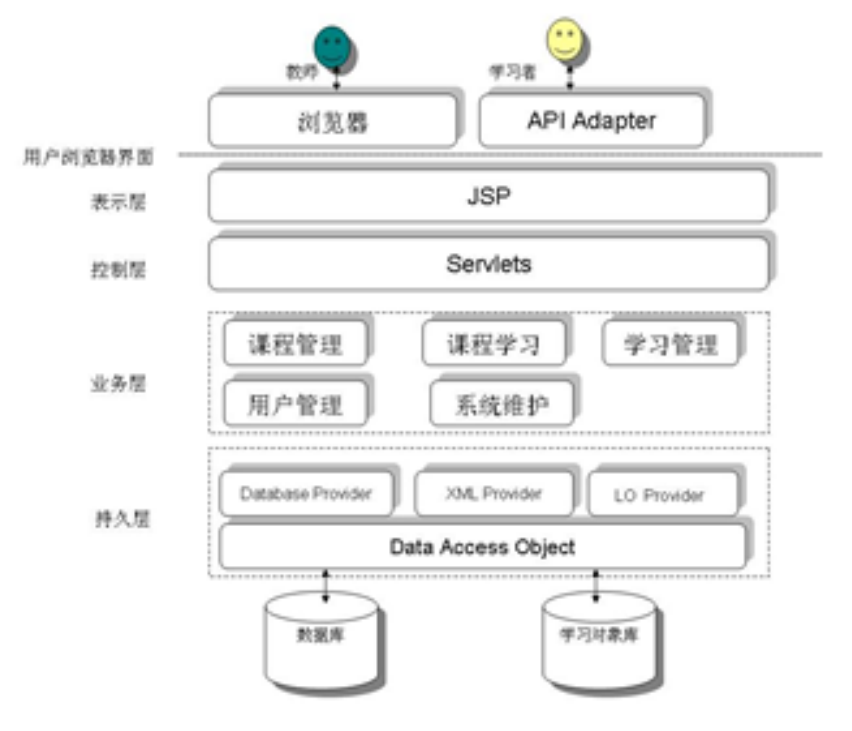

图 3 学习过程跟踪系统架构

整个系统的架构设计过程中, MVC 的思想一直贯穿其 中。系统分为客户端和服务端两部分, 客户端主要采用 Java Applet 技术, 实现 API 适配器。服务器端分为表示层、控 制层、业务层和持久层。其中表示层是系统的人机交互页 面, 定义了浏览器要显示的内容和如何处理用户的请求; 控制层主要实现业务请求转发; 业务层完成事务逻辑的处 理; 持久层用来封装对数据库和学习对象库的访问。

\section{4. 学习过程跟踪关键技术实现}

关键技术包括课件与学习平台交互的 API、学习内容 检索技术、学习记录跟踪与查看等关键技术的实现。

\section{1 学习交互 API 设计与实现}

一个学习对象被启动后, 需要不断的通过 API 与学习 系统进行 Data Model 的交互, 这样势必会增加网络通信的 负担, 并影响系统的性能。解决这个问题的方法就是尽量 减少 API 和 LCMS 之间的交互, 我们的解决办法是在客户 端缓存学习对象所需的 Data Model, 对于 GetValue 和
SetValue 请求, 通过本地接口对客户端缓存的数据进行处 理。这样的话, 学习对象在启动时就需要从服务端获取所 需的数据并缓存到本地, 这个动作是由 Initialize 来完成。 当退出学习对象时, 需要调用 Terminate 来保存这些缓存数 据并更新数据库中的信息。

API 适配器分为本地操作和与服务器端交互两类, 相 应的, 我们也设计了几个辅助类来实现。篇幅有限, 这里 笔者主要阐述一下与服务端交互相关的类, 相关类如图 4 所示。

\begin{tabular}{|c|c|}
\hline SCORM1 3APIInterface & \multirow{6}{*}{ 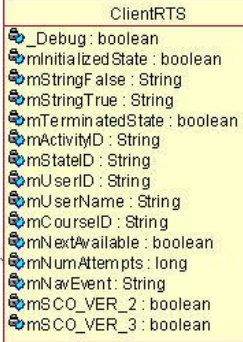 } \\
\hline $\begin{array}{l}\text { \$iParam : String } \\
\text { \$iDataModelElement : String } \\
\text { \$ivalue : String } \\
\text { कiErrorCode : String }\end{array}$ & \\
\hline $\begin{array}{l}\text { Initialize0 } \\
\text { Terminate0 } \\
\text { ogetvalue0 } \\
\text { setvalue0 } \\
\text { commit0 } \\
\text { GetLastErroro }\end{array}$ & \\
\hline ServletProxy & \\
\hline \&_Debug : boolean & \\
\hline postLMSRequest0 & \\
\hline$\hat{n}$ & $\begin{array}{l}\text { "init0 } \\
\text { g get } A p \text { pletinfoo }\end{array}$ \\
\hline Servletwriter & $\begin{array}{l}\text { getParameterlinfor } \\
\text { isInitialized0 }\end{array}$ \\
\hline$\Leftrightarrow$ Debug : boolean & Initialize0 \\
\hline postobjects0 & $\begin{array}{l}\text { Terminateo } \\
\text { Getvalue0 }\end{array}$ \\
\hline APIE rom an ager & - setUistate0 \\
\hline $\begin{array}{l}\text { \$mCurrentErrorCode : int } \\
\text { \$sCORM_1_2_API: int } \\
\text { कSCORM_2004_API : int }\end{array}$ & $\begin{array}{l}\text { - Commito } \\
\text { GetLas Erroro } \\
\text { GetErrorString0 }\end{array}$ \\
\hline $\begin{array}{l}\text { APIErrorManagero } \\
\text { getCurrentErrorCode0 } \\
\text { setCurrentErrorCode0 } \\
\text { clearCurrentErrorCode0 } \\
\text { getErrorDescriptiono } \\
\text { getErrorDiagnostic0 } \\
\text { isValidErrorCode0 }\end{array}$ & $\begin{array}{l}\text { - setActivityD0 } \\
\text { setCourselD0 } \\
\text { setStatelD0 } \\
\text { setUseriD0 } \\
\text { setUserName0 } \\
\text { setNumAttempts0 } \\
\text { "clearState0 }\end{array}$ \\
\hline
\end{tabular}

图 4 API 适配器实现的主要类图

(1) SCORM13APIInterface 类: 提供一个 SCO 和 LCMS 通 信的接口, 该类包含所有在 SCORM 2004 中可以使用 的 API, 该类负责执行所有 LCMS 中 SCORM 2004 定 义的行为。

(2) ClientRTS 类: 这个类是作为一个 applet 在客户端运行 的, 和系统的服务器组件协同工作, 用来处理和存储 数据模型。当前有许多技术可以实现客户端和服务器 端的通信, 但是使用 JavaScript 技术可以把及时通信的 数据缓存到本地, 因此我们选用了这种技术。

(3) ServletProxy 类: 作为 API 适配器与控制层 Servlet 通信 的代理, 将 API 适配器发出对系统的请求对象转化为 序列化对象, 通过调用 ServletWrite 类方法, 将数据发 往控制层 servlet 并获取应答对象流。

(4) ServletWriter 类: 这个类实现了将序列化对象发送到 Servlet, 并从 Servlet 获得序列化对象。这段代码的灵 感来自《Java Servlet Programming》(Jason Hunter 和 William Crawford 编著）这本书。 


\section{2 学习对象检索实现}

进入 SCORM 课程导航模式学习时, 学习者可以通过 表单提交学习对象检索请求, 该功能是在 code.jsp 文件中是 实现的, code.jsp 文件先把关键字的值赋给 str, 然后 code.jsp 文件向持久层发出数据检索的请求, 持久层会在数据表 ItemInfo 中检索符合条件的学习对象, 如果找到数据集则赋 给数组 TOCList, 系统就会根据 TOCList 生成符合条件的学 习对象树形目录。图 5 是学习对象检索的时序图。

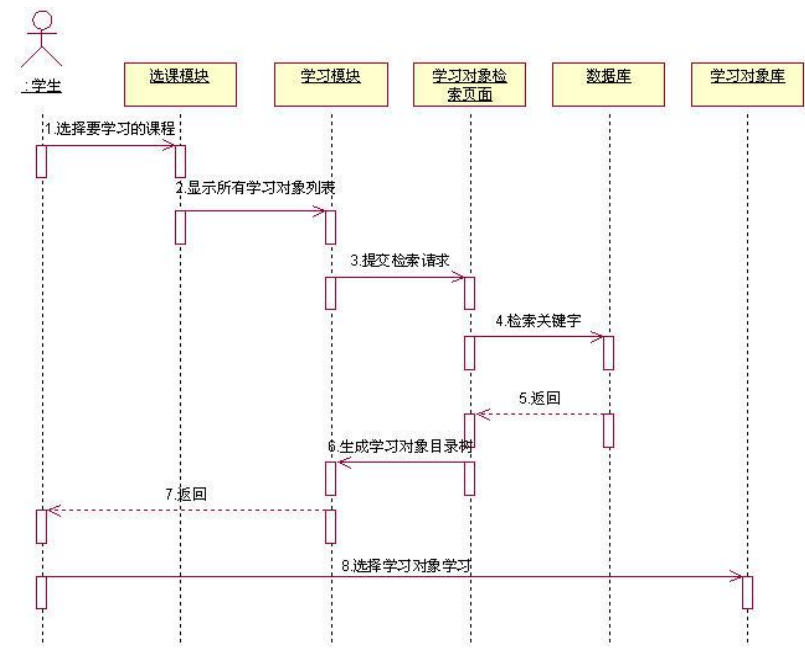

图 5 学习对象检索的时序图

\section{3 查看用户学习记录}

用户学习状态用来记录学生学习课程的进展情况, 当 用户注册一门课程时, 系统就会把选课记录插入到 CourseStatus 数据表中, 对学习状态初始化, 然后在用户学 习课程时, 系统会随时跟踪学习者的学习进展状况, 并通 过类 ADLSeqUtilities 随时更新 CourseStatus 数据表, 记录 学生的学习进展状况, 用来记录学习者学习状态的数据库 字段包括 Learner、Satisfied、Measure、Completed。下面给 出类 ADLSeqUtilities 更新学习者学习状态部分代码实现。

\section{5. 结束语}

面向微课程单元的课程设计方法和网络课程标准化是 未来 E-learning 发展的必然趋势, 而 SCORM 标准作为学习 对象标准的集大成者, 广泛被各国政府和公司采纳。本文 针对现有在线学习平台缺乏有效学习监督、跟踪机制等问 题, 在对学习对象及其标准进行深入研究的基础上, 设计 并实现了符合 SCORM 标准的学习过程跟踪系统, 为提高 的在线学习平台的学习效果做了有益的探索。但 SCORM 标准是一个非常庞大而且复杂的学习对象标准, 学习过程 又是一个非常复杂的过程, 本课题在实现这个系统时无法 做的面面俱到。因此要实现功能更强大的学习过程跟踪系 统还需做更多的工作。

\section{参考文献(References)}

[1] ADL. SCORM 2004 Specification. http://www.adlnet. gov/scorm/ history / 2004/ documents. cfm.

[2] Michael Brennan, Susan Funke, and Cushing Anderson. The Learning Content Management System. http://www. internettime. com/ itimegroup/ lcms/ IDCLCMSWhitePaper.pdf.

[3] Robert Koolen.Learning Content Management Systems- The Second Wave

of eLearning. http://www.internettime.com/Learning/lcms/ lcms2ndwave. pdf

[4] Raghavan Rengarajan. LCMS and LMS Taking Advantage of Tight Integration. http:// www. e-learn.cz/soubory/lcms_and_lms.pdf.

[5] geo. Learning Content Management System Requirements. http://www. edtechpost. ca/ pmwiki/ uploads/Main/Geolearning_LCMS _Requirements. pdf.

[6] Stephen Downes. Learning Objects in a Wider Context. http:// www.downes.ca/ files/widercontext. ppt.

[7] Jason Hunter,William Crawford. Java Servlet Programming. http:// www. cnpoint.com / down /download.php?downid=71\&id=0. 